Original scientific paper

\title{
APPLICATION OF MACHINE LEARNING AND RULE SCHEDULING IN A JOB-SHOP PRODUCTION CONTROL SYSTEM
}

\author{
Zhao, Y. \& Zhang, H." \\ School of Art and Design, Zhejiang Sci-Tech University, Hangzhou 310018, China \\ E-Mail: zy6922@126.com ( ${ }^{\#}$ Corresponding author)
}

\begin{abstract}
As intelligent and precision manufacturing becomes the trend of industrial production, it is of practical significance to study the job-shop production control. However, the existing studies have not provided an evaluation mechanism to reasonably measure the control efficiencies of different plans. The desired control objectives are not easily achieved for job-shop production control problems with dynamic changes. Therefore, this paper probes into the dynamic job-shop production control problem based on deep reinforcement learning and rule scheduling. Firstly, a multi-objective optimization model was established for the production control system of dynamic job-shop. Then, deep reinforcement learning was introduced to job-shop production control system to transform the dynamic job-shop production control problem. After that, the authors proposed a dynamic job-shop production control method based on deep reinforcement learning, and explained the collaboration strategy for multiple subsystems. The proposed method was proved effective through experiments.

(Received in February 2021, accepted in April 2021. This paper was with the authors 2 weeks for 1 revision.)
\end{abstract}

Key Words: Deep Reinforcement Learning, Rule Scheduling, Job-Shop, Production Control

\section{INTRODUCTION}

Job-shop production control is a classic problem in the combinatory optimization of scheduling rules and production control [1-3]. As intelligent and precision manufacturing becomes the trend of industrial production, it is of practical significance to study the job-shop production control. However, the traditional solving algorithms for production control and job-shop scheduling only work under certain application scenarios [4-6]. Meanwhile, the computationalefficient rule scheduling approach cannot fully adapt to the constantly changing production environment and conditions [7-12]. The key and difficulty in the effective application of production control strategy lies in the substantial improvement of control efficiency, and realtime optimization and correction of operations based on existing control information.

Many scholars have explored job-shop production control. For example, Rathore et al. [13] fully considered the constraints on zero waiting of jobs during processing and the operator's learning effect, constructed a mathematical model for zero-waiting flow shop scheduling problem, and solved the model with genetic algorithm (GA) / path relinking search algorithm, which involves elite retention strategy and local search operator. Taking minimal makespan as the object, Lage Junior and Godinho Filho [14] modelled the flow shop scheduling problem with machine allocation, job sequence, and initial processing time as decision variables, and solved the problem with the adaptive GA - symbiotic organisms search (SOS) algorithm, which contains mutualism, commensalism, and parasitism operators. The fitness function of the algorithm determines the adaptability and search effect. Zhang et al. [15] analysed the energy consumption factors of the job-shop, and established an energy consumption calculation model that considers operator factors. Block et al. [16] built up a job-shop production control model to minimize the maximum completion time, production cost, and production energy cost, solved the problem with the non-dominated sorting genetic algorithm II (NSGA-II), which utilizes the 
plug-in greedy decoding operator, and verified the effectiveness of the algorithm by comparing the equilibrium of different production indices under four scheduling plans.

The job-shop production control problem faces constraints on energy consumption and waiting time. The traditional simplified models and solving methods do not work well on such a complex problem. Most of them are overdependent on mathematical models and gradient information [17-19]. Adloor and Vassiliadis [20] improved the differential evolution algorithm, which is easy to implement with a few control parameters, and applied the improved version to solve the energy-efficient production control of zero-waiting flow shop. The improved algorithm successfully activated the current optimal solution, when the global search became stagnant. In addition, Jalilian et al. [21] verified the effectiveness and efficiency of the improved algorithm on production cases.

Facing the dynamic changes of uncertain factors, it is difficult for mathematical modelling, and heuristic and meta-heuristic algorithms to obtain the optimal solution in real time [21-25]. Based on temporal difference (TD) learning theory, Damanik et al. [26] established a deep TD reinforcement learning model, and used the model the transform and solve the multi-phase decision-making of multi-objective flexible job-shop scheduling; the solution was much better than that obtained by heuristic algorithm or ant colony optimization (ACO).

In job-shop production control, there are generally several feasible plans for a problem with multiple objectives and constraints. However, the existing studies have not provided an evaluation mechanism to reasonably measure the control efficiencies of different plans. This paper attempts to realize the desired control objectives of job-shop production control problems with dynamic changes, and narrow the distance between relevant theories and practical problems. Therefore, deep reinforcement learning was combined with rule scheduling, and implemented to solve the multi-objective optimization and rule selection of job-shop production control.

The main contents of this work cover three aspects: (1) setting up a multi-objective optimization model for the production control system of dynamic job-shop; (2) introducing deep reinforcement learning to job-shop production control system, and realizing the transform of the dynamic job-shop production control problem; (3) proposing a dynamic job-shop production control method based on deep reinforcement learning, and explaining the collaboration strategy for multiple subsystems. The proposed method was proved effective through experiments.

\section{MULTI-OBJECTIVE OPTIMIZATION OF DYNAMIC JOB-SHOP PRODUCTION CONTROL}

Considering the changes in the delivery date of products and the material demand in each production link, this paper proposes a multi- objective optimization model for the production control system in dynamic job-shop (Fig. 1). Let $(n, m)$ be the $m^{\text {th }}$ operation of the $n^{\text {th }}$ job in job-shop production process; $S_{1}, S_{2}, \ldots S_{l}$ be the multiple performance indices to be optimized. Then, the objective function $G$ of job-shop production control can be defined as:

$$
G=\min \left(S_{l}\right), l=1,2, \ldots, L
$$

The optimization of $G$ is equivalent to the minimization of $S_{1}, S_{2}, \ldots, S_{l}$. Let $Q_{n m l}$ and $P T_{n m l}$ be the start time and duration of operation $(n, m)$, respectively. During the execution of an independent task, an operation cannot be started before the previous operation is complete. Thus, $G$ needs to satisfy the following constraint:

$$
\text { s.t. } Q_{n m}-Q_{n(m+1)}+F T_{n m} \leq 0, n=1,2, \ldots, N ; m=1,2, \ldots, M
$$

During the execution of an independent task, the start time of the first operation must be equal to or greater than 0 : 


$$
Q_{n 1} \geq 0, n=1,2, \ldots, N
$$

Let $C_{n m l}$ be the binary function about whether operation $(n, m)$ is processed on machine $l$. If yes, $C_{n m l}=1$; otherwise, $C_{n m l}=0$. Since a machine cannot process more than one job at a time, we have:

$$
C_{n m l}=C_{u v l}=1 \text { or } Q_{n m}-Q_{u v}+P T_{n m} \leq 0 \text { or } Q_{u v}-Q_{n m}+P T_{u v} \leq 0, n \neq u
$$

This paper takes the minimal makespan $F T$ and minimal total delay $E T$ as the objectives of job-shop production control, and constructs the penalty function $E F$ of early completion and delay.

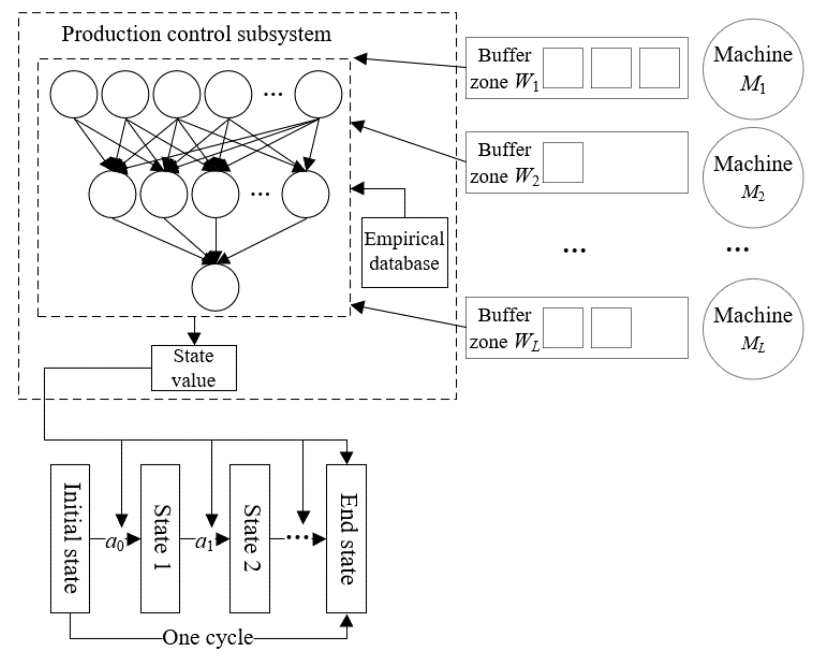

Figure 1: Multi-objective optimization model for the production control system in a dynamic job-shop.

The makespan FT is a key performance indicator of job-shop production control and resource scheduling. Its minimization was set as an optimization objective, that is, minimizing the completion time of the last operation of the last job on all machines. Let $M_{w}$ be the number of jobs to be processed; $F T_{1}, F T_{2}, \ldots, F T_{M w}$ be the completion times of these jobs. Then, the minimization of $F T$ can be expressed as:

$$
\min F T=\min \left(\max \left\{F T_{1}, F T_{2}, . ., F T_{M_{w}}\right\}\right)
$$

Similarly, the minimization of the total delay $E T$ was set as another optimization objective, that is, minimizing the delay of every job on the machines. Let $E T_{i}$ be the delay of the $i^{\text {th }}$ job on the machines. Then, the minimization of ET can be expressed as:

$$
\min E T=\min \sum_{i=1}^{M_{w}}\left\{E T_{i}\right\}
$$

Early completion of job processing will lead to an early delivery, causing inventory waste. Delayed completion will postpone the delivery date, and produce economic losses. To avoid both problems, the penalty function $E F$ was constructed to penalize early completion and delay, such that the processing of every job can be completed around its delivery date. The optimization direction is minimizing the penalty. Let $F T_{i}$ and $D D_{i}$ be the completion time and delivery date of the $i^{\text {th }}$ job, respectively; $E P_{i}$ and $D P_{j}\left(E P_{j}+D P_{j}=1\right.$, and $\left.E P_{j}, D P_{j}>0\right)$ be the penalty coefficients for early completion and delay, respectively. Then, we have:

$$
E F=\left\{\sum_{i=1}^{M_{w}}\left\{\left[E P_{i} \max \left(\left(F T_{i}-D D_{i}\right), 0\right)\right]+\left[D P_{i} \max \left(\left(D D_{i}-F T_{i}\right), 0\right)\right]\right\}\right\}
$$

The sum of $E P_{j}$ and $D P_{j}$ is 1 . The two penalty coefficients are both positive constants.

Next, the $M_{D^{-}}$dimensional vector in the decision space A for dynamic job-shop production control is denoted as $a=\left(a_{1}, a_{2}, \ldots, a_{M D}\right)$, while the $N_{D}$-dimensional vector in the objective 
function $G$ is denoted as $g=\left(g_{1}, g_{2}, \ldots, g_{M}{ }^{D}\right)$. Then, the multi-objective optimization problem for the dynamic job-shop production control with $M_{D}$ decision variables and $N_{D}$ objective variables can be mathematically modelled as:

$$
\min G(x)=\left(g_{1}(a), g_{2}(a), \ldots, g_{M_{D}}(a)\right)
$$

The $u$ inequality constraints $h_{p}(a) \leq 0$ and $M_{D}-u$ equality constraints $f_{q}(a)=0$ can be expressed as:

$$
\text { s.t. }\left\{\begin{array}{l}
h_{p}(a) \leq 0, p=1,2, \ldots, u \\
f_{q}(a)=0, q=u+1, \ldots, M_{D}
\end{array}\right.
$$

Then, the weighted sum of the three objective functions, namely, makespan $F T$, total delay $E T$, and penalty function $E F$, was solved. The result was treated as the multi-objective optimization function for the dynamic job-shop production control problem:

$$
W T=\mu O V+\alpha \psi+\delta F
$$

where, $\mu, \alpha$, and $\delta$ are all positive constants, whose sum equals 1 .

\section{TRANSFORM OF DYNAMIC JOB-SHOP PRODUCTION CONTROL PROBLEM}

Under actual working conditions, job-shop production control faces uncertain explicit disturbances. The common solution is to transform the dynamic control problem in the event of an interference into a static control problem, and to redefine and redesign entity codes, objective functions, resource constraints, and algorithm parameters. This traditional approach is time-consuming, and easily fail with the occurrence of new interferences, calling for manual adjustment. Fortunately, the machine learning algorithm, capable of generating real-time feedbacks to problems, can handle any stochastic event in the process of dynamic job-shop production control at ease.

The dynamic job-shop production control problem can be transformed into a Markov decision process. As shown in Fig. 2, the state space of Markov decision process describes the real-time working conditions required by the job-shop production control system to make decisions about production and processing. In this scenario, the production control system can receive a reward by controlling machines to select and process any job. In this paper, deep reinforcement learning is introduced to the job-shop production control system. By this strategy, the processing task can be executed in such a manner that the machines can receive the highest cumulative reward, after learning a series of production and processing decisions.

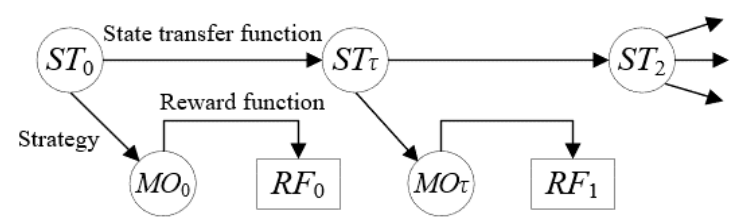

Figure 2: Markov decision process.

Considering the sheer number of states in job-shop production control problem, it is not very feasible to adopt the reinforcement learning based on the entire Markov decision process. Therefore, this paper only chooses the model-free reinforcement learning method that only evaluates the state value. If there are many machines, the action space of only one job-shop production control system is too large for the model, which would greatly complicate the training of reinforcement learning. Therefore, this paper expands the dynamic job-shop production control problem into a Markov decision process of multiple production control subsystems. In this way, multiple production control subsystems are connected with multiple machines, making the problem modelling more flexible and avoiding the redesign of operation sequence in the event of uncertain implicit disturbances. 


\subsection{State space}

Based on deep reinforcement learning, the effect of dynamic job-shop production control depends greatly on the state space, action space, and penalty/reward function of Markov decision process. In this paper, the state space is configured as three matrices: a processing time matrix of all operations, a Boolean matrix of outstanding operations, and a Boolean matrix of completed operations. The rows and columns of the matrices represent different operations and jobs. When a production control subsystem chooses an operation for action in the next state, it must comply with the applicable scheduling rules, in order to respond rapidly to environmental changes. To facilitate the calculation of control objectives in the next step, the above three matrices can be imported as input data to the three input channels of the convolutional neural network $(\mathrm{CNN})$.

\subsection{Action space}

Fig. 3 shows the construction process of action space. In dynamic job-shop production control problem, the action space refers to the actions of every machine selecting any job from the buffer zone for processing under the applicable scheduling rules in the current state. In other words, the job-shop scheduling rules to be followed are the action space of deep reinforcement learning. Fig. 4 shows the execution flows of classic rule scheduling algorithms, including longest processing time (LPT), shortest processing time (SPT), maximum work remaining (MWR), least work remaining (LWR), most operations remaining (MOR), and least operations remaining (LOR).

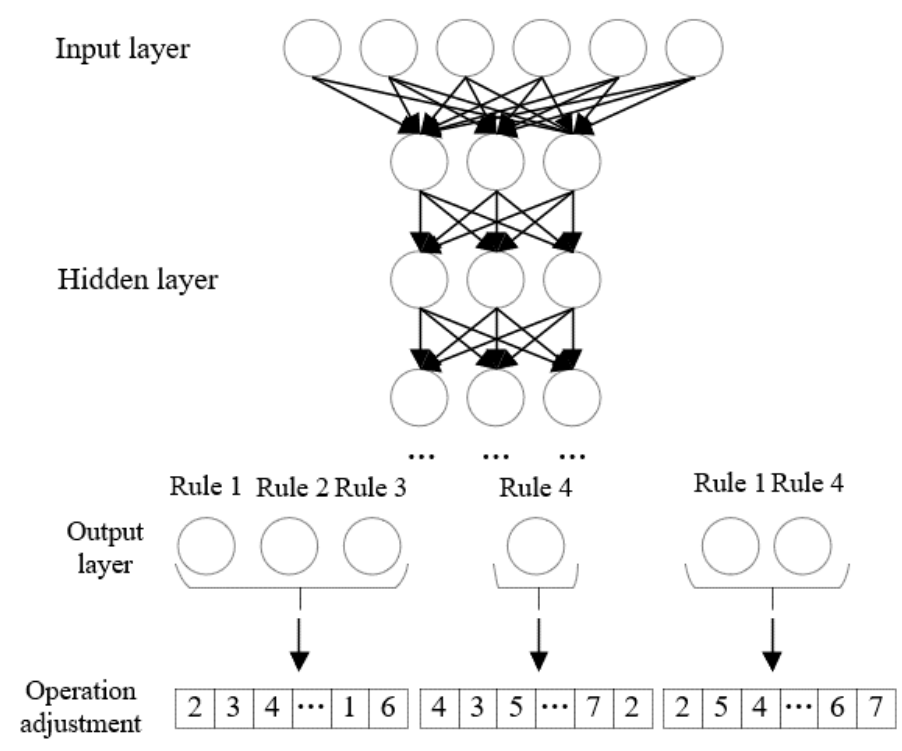

Figure 3: Construction of action space.

\subsection{Penalty/reward function}

This paper defines the penalty/reward function based on the short-term reward of the processing actions by machines. The amount of reward is jointly determined by the processing time of jobs and makespan. Let $\mu$ and $\delta$ be weight coefficients; $b_{O}$ and $b_{P}$ be the distributions of the optimal solution to the makespan and the value predicted by the deep reinforcement learning algorithm, respectively; $\tau_{i}$ be the processing time of the $i^{\text {th }}$ job on machines. Then, the penalty/reward function for machines taking processing action can be defined as:

$$
R F=\frac{\delta}{b_{O}-b_{P}}-\mu \times \tau_{j}
$$




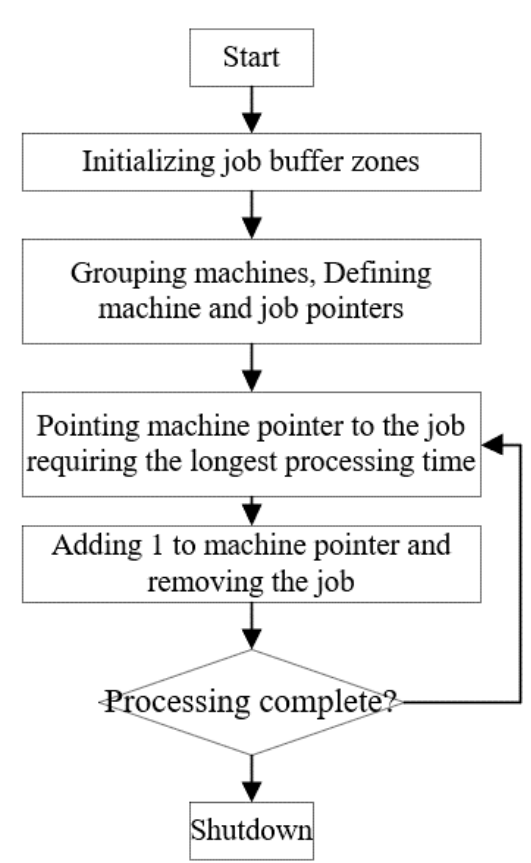

a) LPT

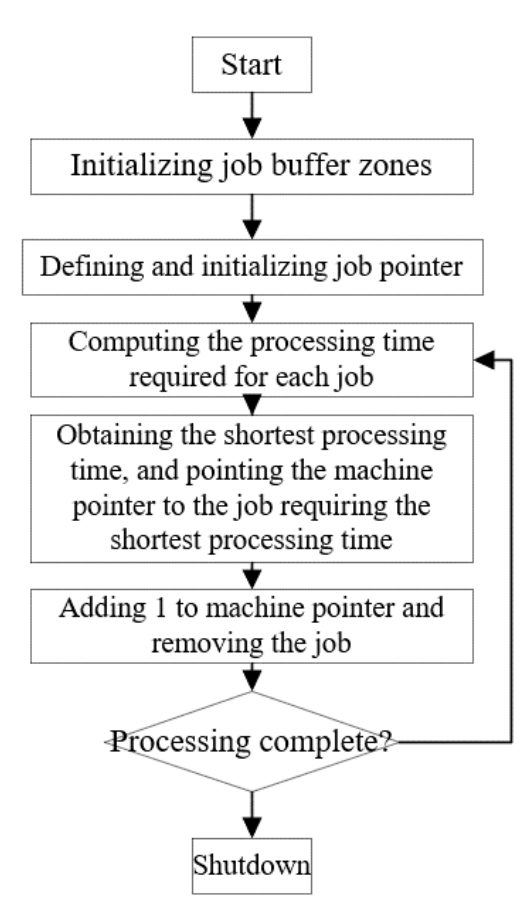

d) LWR

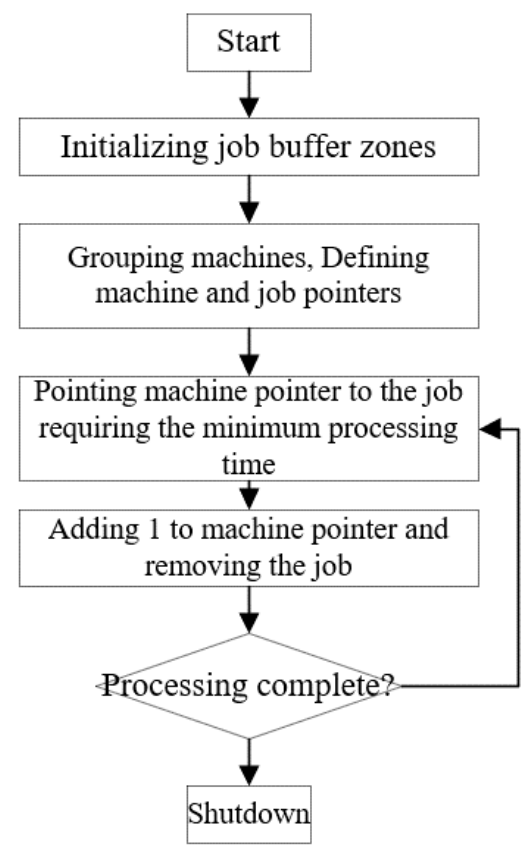

b) SPT

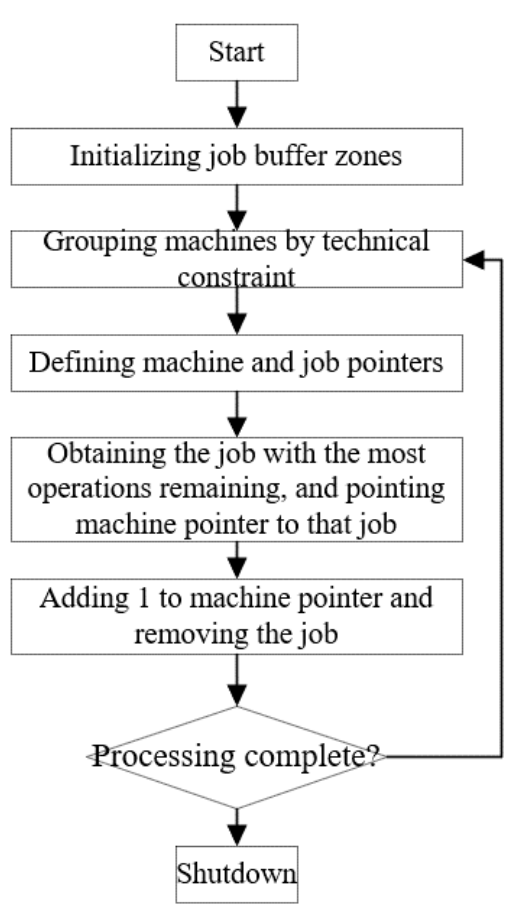

e) MOR

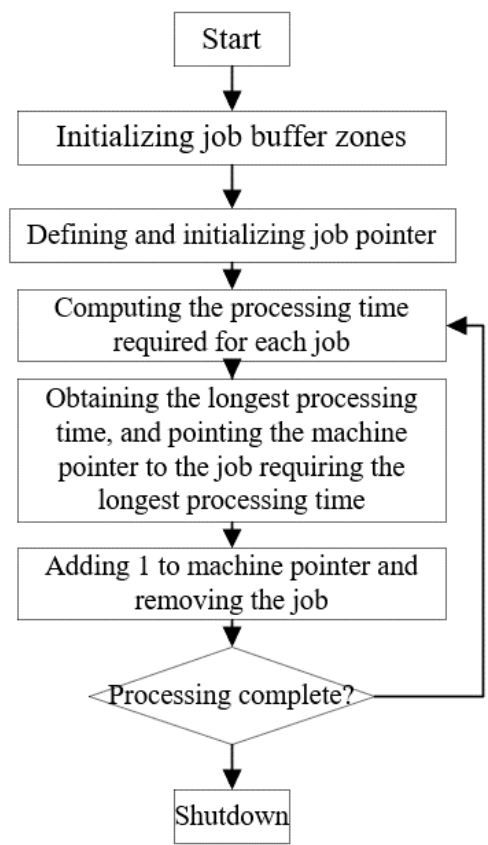

c) MWR

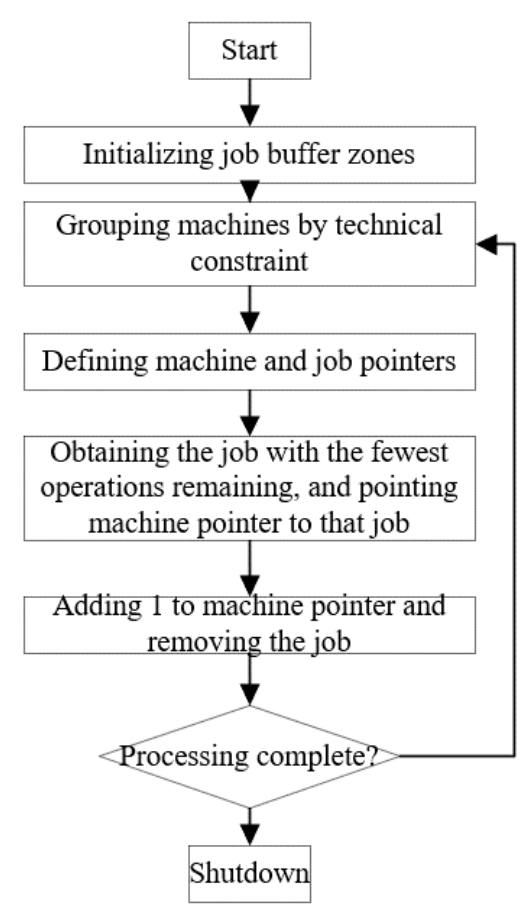

f) LOR

Figure 4: Flowchart of rule scheduling algorithms.

\section{DYNAMIC JOB-SHOP PRODUCTION CONTROL METHOD BASED ON DEEP REINFORCEMENT LEARNING}

\subsection{Model construction}

Based on $\varepsilon$-greedy strategy, this paper explores the processing actions of machines in the process of dynamic job-shop production control. A random number from uniform distribution was compared with the pre-set exploration threshold $\beta$ to check if it is greater than that threshold. 
Let $V_{O}$ be the size of the action space. If $a>\beta$, the processing action $M O(\tau)$ of a machine can be determined by the action exploration strategy:

$$
M O(\tau) \leftarrow O\left(0, V_{o}\right)
$$

If $M O_{\tau}=0$, the machine controlled by the production control subsystem has no action to execute; if $M O_{\tau}=V_{O}$, the machine controlled by the production control subsystem executes production action $V_{O}$. Let $\Phi(M O \mid S T)$ be the action strategy of the machines participating in production. If $a<\beta$, action $M O(\tau)$ can be determined by greedy strategy:

$$
M O(\tau) \leftarrow \arg \max \Phi(M O \mid S T)
$$

The deep reinforcement learning network is activated by rectified linear unit $(\operatorname{Re} L U)$ function:

$$
\operatorname{Re} L U(a)=\left\{\begin{array}{l}
a,(a>0) \\
0,(a \leq 0)
\end{array}\right.
$$

If the input to $\operatorname{Re} L U$ is negative, the network layer will output zero; if the input is positive, the input will be directly outputted. $\operatorname{Re} L U$ function does better than Sigmoid and Tanh in network stabilization and unilateral inhibition.

For largescale job-shop production control problem, the median function of Q-learning has a large capacity. Based on deep Q-learning network, this paper simultaneously estimates the state values and action values of production control.

During the learning and training of the production control subsystems in a dynamic environment, the real-time update efficiency of each subsystem will be reduced due to the strong correlation between the state at time $\tau$ and that at time $\tau+k$.

This issue can be easily solved by the large empirical data pool of the deep learning algorithm based on experience replay mechanism. Based on deep learning algorithm, the empirical steps of subsystems were randomly sampled to replace the traditional real-time update.

To sum up, this paper analyses job-shop scheduling problem from two aspects, namely, action implementation and action evaluation, with production control subsystems containing Actor network and Critic network.

\subsection{Loss function and parameter update}

Fig. 5 shows the structure of the loss function for our model. It can be seen that the deep Qlearning network is updated by a loss function composed of two parts: Actor loss and Critic loss. Let $\omega_{c r}$ be the parameter of the Critic network; $p$ be the instant reward obtained by a machine; $Q\left(S T^{*}, M O^{*}, \omega_{c r}\right)$ be the estimated value of the next production state of the machine. Then, the Critic loss function can be given by:

$$
\operatorname{Loss}_{c r}\left(\omega_{c r}\right)=\left(p+\delta \max Q\left(S T^{*}, M O^{*}, \omega_{c r}\right)-Q\left(S T, M O, \omega_{c r}\right)\right)^{2}
$$

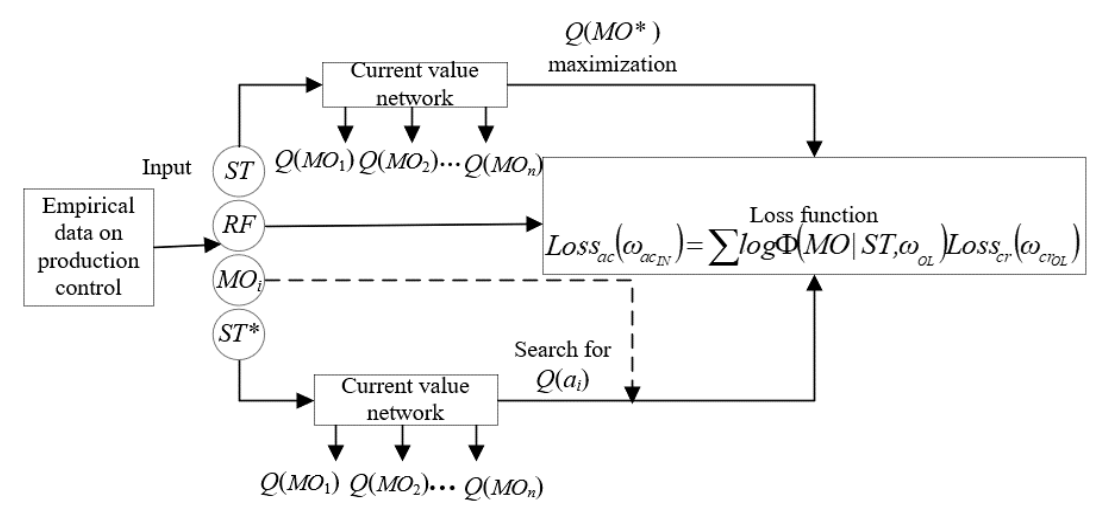

Figure 5: Structure of loss function. 
The Critic loss function is updated by the same strategy as the Q-learning network based on value function. The update aims to minimize the gap between the estimated values of machine state and action functions $Q\left(B E^{*}, M O^{*}, \omega_{c r}\right)$ and the target values $p+\delta_{\max } Q\left(B E^{*}, M O^{*}, \omega_{c r}\right)$. Let $\omega_{a c}$ be the parameter of the Actor network. Then, the Actor loss function can be given by:

$$
\text { Loss }_{a c}\left(\omega_{a c}\right)=\sum \log \Phi\left(M O \mid S T, \omega_{M O}\right) \text { Loss }_{c r}\left(\omega_{c r}\right)
$$

Eq. (16) shows that, if a production subsystem is unlikely to control machines to execute a production task, yet receives a high reward, then the $\operatorname{Loss}_{a c}\left(\omega_{a c}\right)$ will increase. Fig. 6 shows the update pattern of the network model.

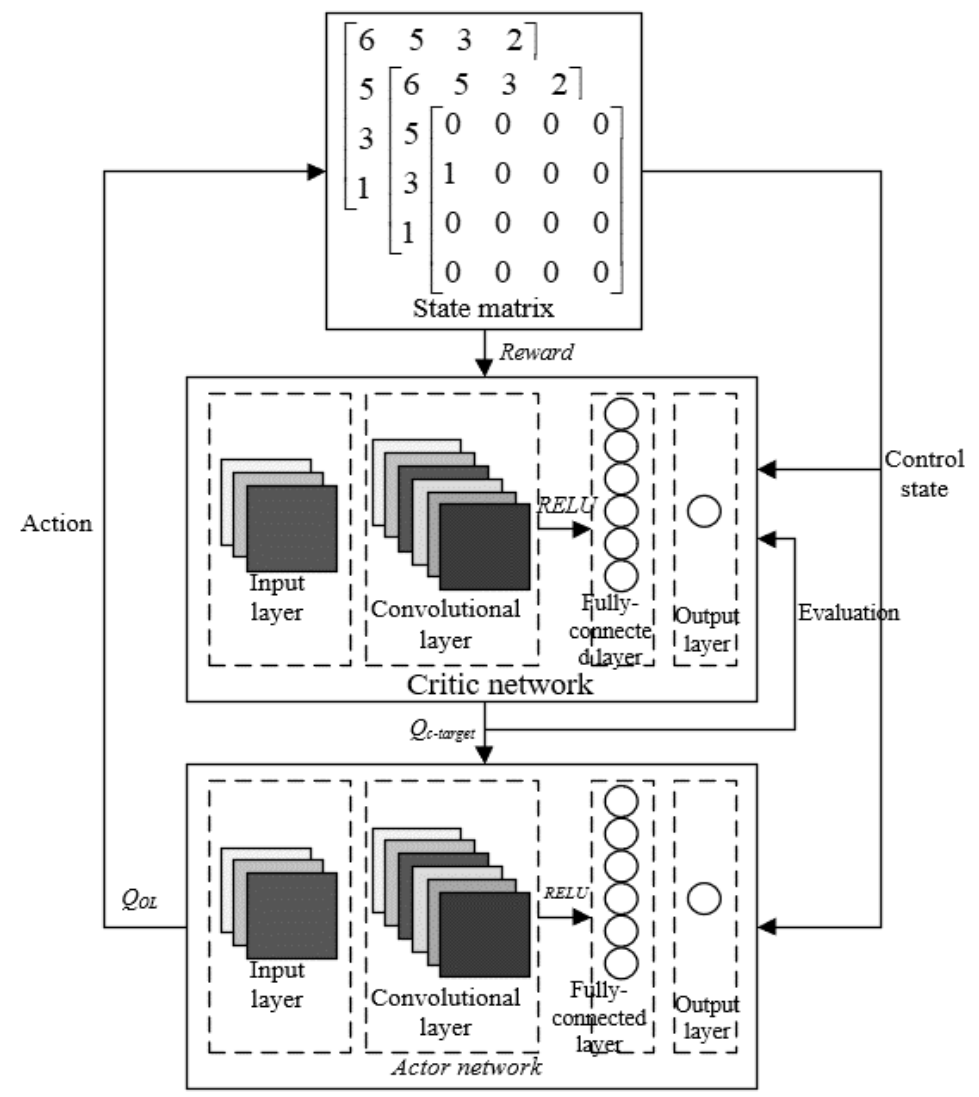

Figure 6: Update pattern of online model.

If the Critic has disagreement, the delayed target network needs to be introduced to the algorithm. Then, the Critic loss function of online network can be updated by:

$$
\operatorname{Loss}_{c r}\left(\omega_{c r_{L L}}\right)=\left(p+\delta \max _{M O_{T}^{*}} Q\left(S T, M O^{*}, \omega_{c r_{T}}\right)-Q\left(S T, M O, \omega_{c r_{T}}\right)\right)^{2}
$$

where, the second bracketed term on the right side indicates that Critic will evaluate the action from the delayed target network in state $S T^{*}$. Similarly, the Actor loss function can be updated by:

$$
\operatorname{Loss}_{a c}\left(\omega_{a c_{N}}\right)=\sum \log \Phi\left(M O \mid S T, \omega_{o L}\right) \operatorname{Loss}_{c r}\left(\omega_{c r_{L L}}\right)
$$

In each iteration, the parameters can be configured as:

$$
\begin{aligned}
& \omega_{a c_{T}}=\omega_{a c_{O L}} \\
& \omega_{c_{T}}=\omega_{c r_{O L}}
\end{aligned}
$$

\subsection{Collaborative control of multiple production control subsystems}

For a largescale dynamic job-shop production control system, the model construction involves multiple subsystems. Whenever a subsystem takes an action, its state changes will affect the 
actions and states of other subsystems. Therefore, the primary task of dynamic job-shop production control system is to update and coordinate every subsystem. Fig. 7 illustrates the learning and training pattern of multiple production control subsystems. Each subsystem that controls machines copies its parameters from the global network. The network training for each subsystem is arranged according to the actual production environment. Then, the global system updates parameters according to the cumulative gradients of all subsystems.

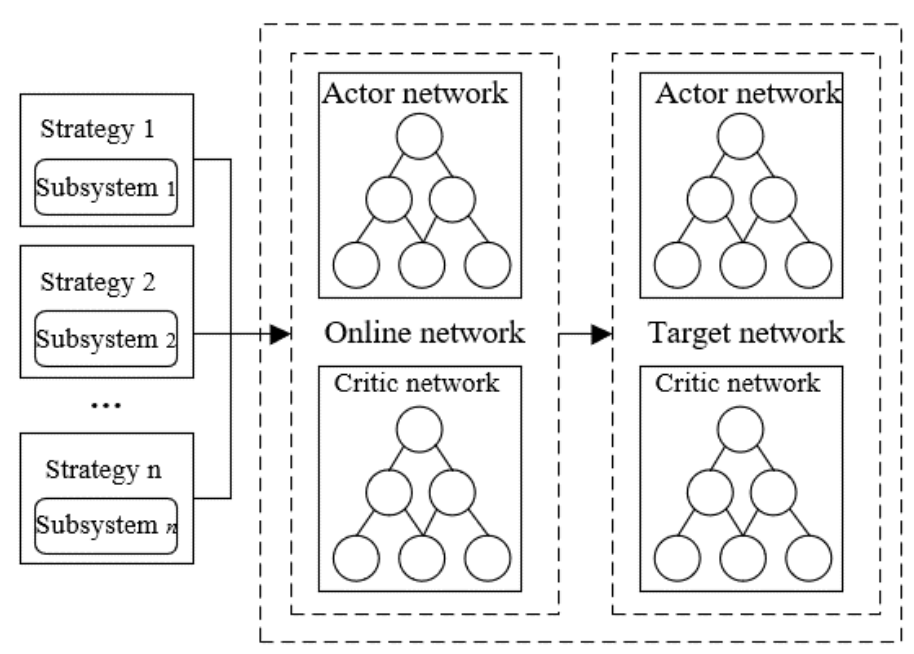

Figure 7: Learning and training pattern of multiple production control subsystems.

\section{EXPERIMENTS AND RESULTS ANALYSIS}

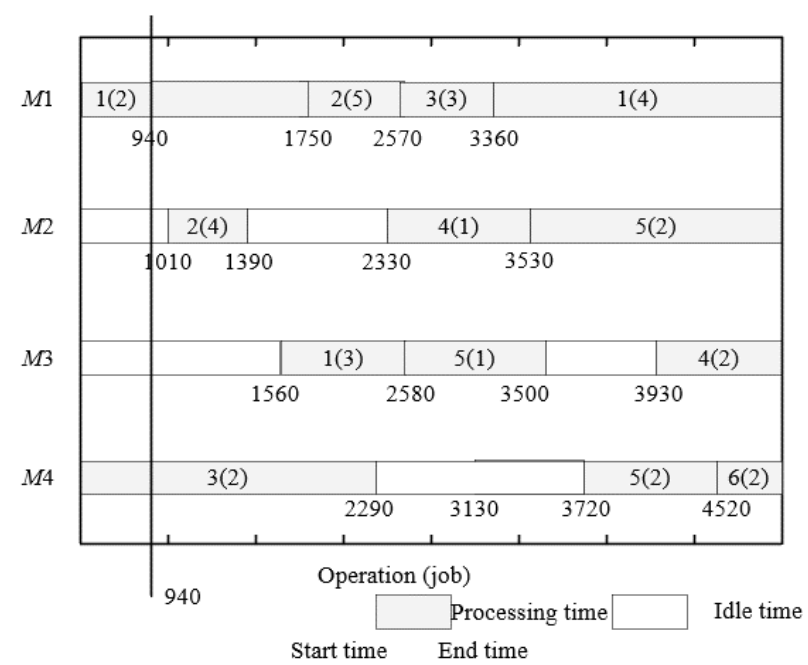

a) GA

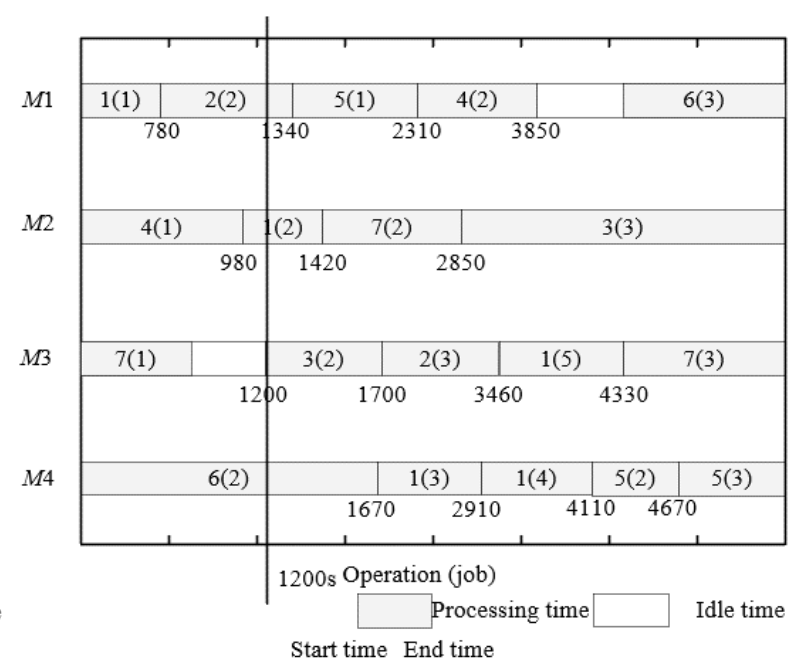

b) Machine learning algorithm.

Figure 8: Gantt charts of production control.

Fig. 8 provides the Gantt charts of production control of traditional GA and machine learning algorithm. The simulation results show that the scheduling rules for production control plans can be ranked as SPT, LPT, MWR, LWR, MOR, LOR, based on the priority derived from deep reinforcement learning coupled with rule scheduling. The Gantt charts show that, in the production control plan of the optimal solution obtained by our algorithm, after the completion of each operation, the corresponding machine needs to execute one scheduling rule adaptation, until all the jobs are processed.

To further verify the effectiveness of the proposed dynamic job-shop production control algorithm, which integrates deep reinforcement learning network with rule scheduling, this paper summarizes the weights of objective functions and $E F$ coefficients from different 
production items (Table I). Table II records the adaptation to six scheduling rules during the modelling process.

Table I: Weights of objective functions and $E F$ coefficients.

\begin{tabular}{|c|c|c|c|c|c|}
\cline { 2 - 6 } \multicolumn{1}{c|}{} & \multicolumn{2}{c|}{ Weights of objective functions } & \multicolumn{2}{c|}{$E F$ coefficients } \\
\hline Item number & $\mu$ & $\alpha$ & $\delta$ & $E P_{i}$ & $D P_{j}$ \\
\hline 1 & 0.1 & 0.5 & 0.2 & 0.5 & 0.7 \\
\hline 2 & 0.3 & 0.4 & 0.5 & 0.7 & 0.9 \\
\hline 3 & 0.3 & 0.3 & 0.4 & 0.3 & 0.4 \\
\hline 4 & 0.5 & 0.2 & 0.5 & 0.2 & 0.3 \\
\hline 5 & 0.7 & 0.1 & 0.3 & 0.3 & 0.5 \\
\hline 6 & 0.6 & 0.9 & 0.5 & 0.1 & 0.1 \\
\hline 7 & 0.4 & 0.6 & 0.2 & 0.9 & 0.5 \\
\hline 8 & 0.5 & 0.4 & 0.3 & 0.2 & 0.6 \\
\hline 9 & 0.2 & 0.2 & 0.2 & 0.7 & 0.3 \\
\hline 10 & 0.5 & 0.5 & 0.2 & 0.9 & 0.4 \\
\hline
\end{tabular}

Table II: Adaptation to six scheduling rules during the modelling process.

\begin{tabular}{|c|c|c|c|c|c|}
\hline Scheduling rule & Waiting time & Number of delays & Mean queue length & Machine utilization & $F T$ \\
\hline SPT & 57 & 1 & 1.32 & 0.35 & 63 \\
\hline LPT & 78 & 1 & 1.43 & 0.36 & 70 \\
\hline MWR & 82 & 1 & 1.45 & 0.31 & 72 \\
\hline LWR & 58 & 1 & 1.33 & 0.37 & 63 \\
\hline MOR & 75 & 1 & 1.42 & 0.38 & 71 \\
\hline LOR & 55 & 1 & 1.47 & 0.32 & 73 \\
\hline
\end{tabular}

Table III: Rule combination and objective function value for the optimal solution in each period.

\begin{tabular}{|c|c|c|c|c|c|}
\hline Period & Rule combination & $F T$ & $E T$ & $E F$ & Objective function value \\
\hline 1 & SPT, MWR, LOR & 58.00 & 19.00 & 22.00 & 18.35 \\
\hline 2 & SPT, LWR, MOR & 58.00 & 8.00 & 26.00 & 27.14 \\
\hline 3 & SPT, LWR, MOR & 58.00 & 18.00 & 16.00 & 45.80 \\
\hline 4 & SPT, MWR, MOR & 58.00 & 5.00 & 27.60 & 37.50 \\
\hline 5 & LPT, MWR, MWR & 58.00 & 3.00 & 33.00 & 32.50 \\
\hline 6 & LPT, LWR, MOR & 58.00 & 2.00 & 51.25 & 41.75 \\
\hline 7 & LPT, LWR, MWR & 58.00 & 22.00 & 14.00 & 47.96 \\
\hline 8 & LPT, MWR & 58.00 & 7.00 & 17.65 & 38.25 \\
\hline 9 & LPT, LWR, MWR & 58.00 & 15.00 & 17.80 & 29.37 \\
\hline 10 & LPT, LWR, LWR & 58.00 & 13.00 & 18.20 & 28.16 \\
\hline
\end{tabular}

Table III presents the rule combination, the value of each objective function, and the value of total objective function in each of the ten research periods, corresponding to the optimal solution of dynamic job-shop production control problem. It can be seen that the same production control plan could apply to different production items and periods; the only difference lies in the sequence of scheduling rules within the rule combination.

Table IV: Execution results of the production control plans by different algorithms.

\begin{tabular}{|c|c|c|c|c|c|}
\hline Model & Waiting time & Number of delays & Mean queue length & Machine utilization & $F T$ \\
\hline GA & 55 & 1 & 1.36 & 0.37 & 66 \\
\hline Our algorithm & 51 & 1 & 1.45 & 0.39 & 53 \\
\hline
\end{tabular}

Table IV displays the execution results of the production control plans by different algorithms. It can be seen that the production control plan generated by our algorithm achieved better effect than that produced by GA. This means the scheduling can be optimized by calling the deep reinforcement learning algorithm. 


\section{CONCLUSIONS}

Based on deep reinforcement learning and rule scheduling, this paper investigates the dynamic job-shop production control problem. Specifically, a multi-objective optimization model was created for the production control system of dynamic job-shop. Then, deep reinforcement learning was introduced to job-shop production control system to transform the dynamic jobshop production control problem. This is followed by the proposal of a dynamic job-shop production control method based on deep reinforcement learning, and the explanation of the collaboration strategy for multiple subsystems. Through experiments, the Gantt chart on the production control by traditional GA was compared with that produced by machine learning algorithm; the rule combination and objective function value were obtained for the optimal solution in each period; the execution results of the production control plans by different algorithms were summarized. The results fully demonstrate the effectiveness of the proposed dynamic job-shop production control algorithm, which couples deep reinforcement learning network and rule scheduling.

\section{ACKNOWLEDGEMENT}

The paper was supported by "Scientific Research Start-Up Fund of Zhejiang Sci-Tech University, China" (Grant No.: 19082415-Y).

\section{REFERENCES}

[1] Satyro, W. C.; de Mesquita Spinola, M.; de Almeida, C. M. V. B.; Giannetti, B. F.; Sacomano, J. B.; Contador, J. C.; Contador, J. L. (2021). Sustainable industries: Production planning and control as an ally to implement strategy, Journal of Cleaner Production, Vol. 281, Paper 124781, 11 pages, doi:10.1016/j.jclepro.2020.124781

[2] Zhang, Z.; Guan, Z. L.; Zhang, J.; Xie, X. (2019). A novel job-shop scheduling strategy based on particle swarm optimization and neural network, International Journal of Simulation Modelling, Vol. 18, No. 4, 699-707, doi:10.2507/IJSIMM18(4)CO18

[3] Gocken, T.; Dosdogru, A. T.; Boru, A.; Gocken, M. (2019). Integrating process plan and part routing using optimization via simulation approach, International Journal of Simulation Modelling, Vol. 18, No. 2, 254-266, doi:10.2507/IJSIMM18(2)470

[4] Beloglazov, I.; Petrov, P.; Martynov, S. (2018). Application of production processes control algorithm using adaptive control system, Proceedings of the 2018 International Russian Automation Conference, 4 pages, doi:10.1109/RUSAUTOCON.2018.8501801

[5] Ren, J.; Ye, C.; Li, Y. (2020). A two-stage optimization algorithm for multi-objective job-shop scheduling problem considering job transport, Journal Européen des Systèmes Automatisés, Vol. 53, No. 6, 915-924, doi:10.18280/jesa.530617

[6] Haronian, E.; Sacks, R. (2018). A specialized information schema for production planning and control of road construction, Karlshoj, J.; Scherer, R. (Eds.), eWork and eBusiness in Architecture, Engineering and Construction, CRC Press, London, 257-264, doi:10.1201/9780429506215

[7] He, D.; Wang, Z.; Yang, L.; Mao, Z. (2016). Optimization control of the color-coating production process for model uncertainty, Computational Intelligence and Neuroscience, Vol. 2016, Paper 9731823, 12 pages, doi:10.1155/2016/9731823

[8] Cangea, O.; Bucur, G.; Popescu, C.; Moise, A. G.; Vlas, D. (2016). Simulation of high quality fish oil production monitoring and control winterization process, Revista de Chimie, Vol. 67, No. 5, 943-947

[9] Andonovski, G.; Mušič, G.; Blažič, S.; Škrjanc, I. (2016). On-line evolving cloud-based model identification for production control, IFAC-PapersOnLine, Vol. 49, No. 5, 79-84, doi:10.1016/ j.ifacol.2016.07.093

[10] Weinberg, M.; Meuser, H. (2016). Integrated process-and quality control to secure the production of slabs for plates for line pipe application, Proceedings of the $11^{\text {th }}$ International Pipeline Conference, Paper V003T05A019, 7 pages, doi:10.1115/IPC2016-64099 
[11] Orihuela, P.; Orihuela, J.; Pacheco, S. (2016). Information and communications technology in construction: A proposal for production control, Procedia Engineering, Vol. 164, 150-157, doi:10.1016/j.proeng.2016.11.604

[12] Pirhanov, A.; Bridges, C. M.; Goodwin, R. A.; Guo, Y.-S.; Furrer, J.; Shor, L. M.; Gage, D. J.; Cho, Y. K. (2021). Optogenetics in sinorhizobium meliloti enables spatial control of exopolysaccharide production and biofilm structure, ACS Synthetic Biology, Vol. 10, No. 2, 345 356, doi:10.1021/acssynbio.0c00498

[13] Rathore, A. S.; Nikita, S.; Thakur, G.; Deore, N. (2021). Challenges in process control for continuous processing for production of monoclonal antibody products, Current Opinion in Chemical Engineering, Vol. 31, Paper 100671, 10 pages, doi:10.1016/j.coche.2021.100671

[14] Lage Jr., M.; Godinho Filho, M. (2016). Production planning and control for remanufacturing: exploring characteristics and difficulties with case studies, Production Planning \& Control, Vol. 27, No. 3, 212-225, doi:10.1080/09537287.2015.1091954

[15] Zhang, H.; Tang, D.; Zheng, K.; Giret, A. (2018). Production control strategy inspired by neuroendocrine regulation, Proceedings of the Institution of Mechanical Engineers, Part B: Journal of Engineering Manufacture, Vol. 232, No. 1, 67-77, doi:10.1177/0954405416639889

[16] Block, C.; Lins, D.; Kuhlenkötter, B. (2018). Approach for a simulation-based and event-driven production planning and control in decentralized manufacturing execution systems, Procedia CIRP, Vol. 72, 1351-1356, doi:10.1016/j.procir.2018.03.204

[17] Altenmüller, T.; Stüker, T.; Waschneck, B.; Kuhnle, A.; Lanza, G. (2020). Reinforcement learning for an intelligent and autonomous production control of complex job-shops under time constraints, Production Engineering, Vol. 14, 319-328, doi:10.1007/s11740-020-00967-8

[18] Dittrich, M.-A.; Fohlmeister, S. (2020). Cooperative multi-agent system for production control using reinforcement learning, CIRP Annals, Vol. 69, No. 1, 389-392, doi:10.1016/ j.cirp.2020.04.005

[19] Mu, H. (2019). Disruption management of flexible job shop scheduling considering behavior perception and machine fault based on improved NSGA-II algorithm, Journal Européen des Systèmes Automatisés, Vol. 52, No. 2, 149-156, doi:10.18280/jesa.520206

[20] Adloor, S. D.; Vassiliadis, V. S. (2020). An optimal control approach to scheduling maintenance and production in parallel lines of reactors using decaying catalysts, Computers \& Chemical Engineering, Vol. 142, Paper 107025, 23 pages, doi:10.1016/j.compchemeng.2020.107025

[21] Jalilian, N.; Najafpour, G. D.; Khajouei, M. (2020). Macro and micro algae in pollution control and biofuel production - a review, ChemBioEng Reviews, Vol. 7, No. 1, 18-33, doi:10.1002/cben.201900014

[22] Yue, H.; Xing, K.; Hu, H.; Wu, W.; Su, H. (2019). Supervisory control of deadlock-prone production systems with routing flexibility and unreliable resources, IEEE Transactions on Systems, Man, and Cybernetics: Systems, Vol. 50, No. 10, 3528-3540, doi:10.1109/ TSMC.2019.2917882

[23] Assid, M.; Gharbi, A.; Hajji, A. (2020). Production control of failure-prone manufacturingremanufacturing systems using mixed dedicated and shared facilities, International Journal of Production Economics, Vol. 224, Paper 107549, 18 pages, doi:10.1016/j.ijpe.2019.107549

[24] Unterberger, E.; Hofmann, U.; Min, S.; Glasschröder, J.; Reinhart, G. (2018). Modeling of an energy-flexible production control with SysML, Procedia CIRP, Vol. 72, 432-437, doi:10.1016/j.procir.2018.03.111

[25] Lalwani, M. A.; Ip, S. S.; Carrasco-Lopez, C.; Day, C.; Zhao, E. M.; Kawabe, H.; Avalos, J. L. (2021). Optogenetic control of the lac operon for bacterial chemical and protein production, Nature Chemical Biology, Vol. 17, No. 1, 71-79, doi:10.1038/s41589-020-0639-1

[26] Damanik, F. F. R.; Verkoelen, N.; van Blitterswijk, C.; Rotmans, J.; Moroni, L. (2021). Control delivery of multiple growth factors to actively steer differentiation and extracellular matrix protein production, Advanced Biology, Vol. 5, No. 4, Paper 2000205, 12 pages, doi:10.1002/ $\underline{\text { adbi.202000205 }}$ 